\title{
Henri Ponchon, L'Incroyable Saga des Torlonia, des Monts du Forez aux Palais romains
}

\section{Michel Arrous}

\section{(2) OpenEdition}

1 Journals

\section{Édition électronique}

URL : http://journals.openedition.org/studifrancesi/9577

DOI : 10.4000/studifrancesi.9577

ISSN : 2421-5856

Éditeur

Rosenberg \& Sellier

\section{Édition imprimée}

Date de publication : 1 décembre 2007

Pagination : 670-671

ISSN : 0039-2944

\section{Référence électronique}

Michel Arrous, « Henri Ponchon, L'Incroyable Saga des Torlonia, des Monts du Forez aux Palais romains », Studi Francesi [En ligne], 153 (LI | III) | 2007, mis en ligne le 30 novembre 2015, consulté le 08 janvier 2021. URL : http://journals.openedition.org/studifrancesi/9577 ; DOI : https://doi.org/10.4000/ studifrancesi.9577

Ce document a été généré automatiquement le 8 janvier 2021.

\section{(c)}

Studi Francesi è distribuita con Licenza Creative Commons Attribuzione - Non commerciale - Non opere derivate 4.0 Internazionale. 


\title{
Henri Ponchon, L'Incroyable Saga des Torlonia, des Monts du Forez aux Palais romains
}

\author{
Michel Arrous
}

\section{RÉFÉRENCE}

HENRI PONCHON, L'Incroyable Saga des Torlonia, des Monts du Forez aux Palais romains, Olliergues, Éditions de la Montmarie, 2005, pp. 344.

1 Si les lecteurs de Rome, Naples et Florence, des Promenades dans Rome et de Vanina Vanini, comme ceux du Comte de Monte-Cristo, connaissent les réceptions fastueuses que Giovanni Torlonia, «riche banquier fort juif», «fort avare et un peu fripon», au jugement de Stendhal qui pensa un instant le prendre comme modèle de l'enrichi dans Lamiel, donnait dans son palais de la place de Venise, sans doute ignorent-ils sa fabuleuse histoire et celle de la dynastie qu'il fonda. Les stendhaliens, qui ont lu la brève étude d'Aimé Dupuy («Stendhal club», 15 octobre 1968) et parcouru la correspondance du consul, disposeront désormais du solide et agréable ouvrage de référence qu'Henri Ponchon a consacré aux Torlonia, les Rothschild de Rome, objet de nombreuses études en Italie, mais rarement évoqués en France: Henri Pourrat a consacré quelques lignes de son Gaspard des montagnes au fondateur de la lignée; Jean Anglade l'a évoqué dans un roman historique, Qui t'a fait prince? (1992). Giovanni Torlonia (1754-1829) est le petit-fils d'un fort modeste marchand de toiles du Forez, Antoine Tourlonias, et le fils de Marin Tourlonias (1725-1785) qui s'installa à Rome en 1750, où il italianisa son patronyme en Torlonia. Après un détour nécessaire chez les Tourlonias du Forez, famille de forgerons et de marchands, H. Ponchon, généalogiste émérite, tente de reconstituer l'étonnant parcours de Marin qui se serait donc fixé à Rome, au service d'une de ses relations familiales, l'abbé de Montgon, agent de Philippe $\mathrm{V}$ d'Espagne, lequel eut de si sévères démêlés avec le cardinal de Fleury qu'il préféra se réfugier dans le palais Zuccari, tout près de la Trinité des Monts, où séjournèrent 
Reynolds, les Nazaréens, et même le grand Winckelmann. D'abord valet de chambre puis marchand drapier, Marino épouse la fille d'un émigré français et d'une notable allemande. Le couple aura quinze enfants dont Giovanni, «ce fameux marchand de fil» (Stendhal), le héros de la famille, qui fondera la dynastie princière des Torlonia, avec l'aide de son frère Giuseppe. Le commerce prospère tant et si bien que les Torlonia se consacrent à la banque. Quoique Giovanni n'ait pas été immédiatement accepté dans le corps des banquiers romains, il réussit à faire de sa maison la première sur la place de Rome. Son fils Alessandro lui succédera et, de 1829 jusqu'en 1860, dirigera la banque qui sera vendue en 1869 et mise en liquidation en 1872.

2 Le chapitre III retrace l'«irrésistible ascension» de Giovanni qui sut profiter des bouleversements provoqués par la Révolution française: banquier de la papauté (qui le fera marquis puis duc), mais aussi fournisseur des armées de la République, approvisionneur de la République romaine, banquier de tous les Bonaparte et de la noblesse romaine, représentant à Rome du prince de Fürstenberg (qui le fera noble d'Empire en 1794), et chargé des intérêts de la Pologne, etc... Une telle réussite suppose bien évidemment des capitaux disponibles et une grande intelligence financière. La banque Torlonia est comparable à celle des Rothschild, mais de moindre envergure: d'abord des opérations de change et l'utilisation de capitaux d'origine commerciale inemployés, puis l'acceptation d'effets émis en Europe par la papauté. Au long des vingt années de conflits entre la France et le Saint-Siège, Torlonia sera présent à toutes les étapes, naviguant entre la papauté et les gouvernements que la France lui impose. Son nom apparaît fréquemment dans les dépêches françaises, notamment lors de l'assassinat de Basseville, l'imprudent secrétaire d'ambassade dont Stendhal a raconté la fin tragique d'après Monti, et dans les rapports de Cacault quand la France imposa au pape l'armistice de Bologne (1796). Giovanni devient alors le banquier d'un pape qui n'a pas assez d'argent pour payer la contribution d'armistice. Comme la France accepte d'être payée en fournitures, de l'alun notamment (qui avait fait la fortune des Chigi au $\mathrm{xvi}^{\mathrm{e}}$ siècle), il va en assurer le transport par Civitavecchia. Après le traité de Tolentino (1797), notre homme intervient encore en signant de nombreuses lettres de change pour le pape; il signe même un compromis avec la France. Il poursuit son ascension en participant avec beaucoup d'habileté et un peu de chance à des opérations toujours juteuses en période troublée: fournitures pour les armées, approvisionnement de la ville de Rome, achat de biens nationaux, participations financières diverses (tissages, bois, etc...). La banque Torlonia est une des plus solides et des plus prospères et, à la chute de la République, Giovanni se retrouve propriétaire d'immenses domaines entre Rome et la mer. Son principe: "Crescere a dismisura». À la fin du siècle, du fait de l'explosion des bénéfices entre 1797 et 1800, sa fortune est faite; il est considéré comme le plus riche banquier de l'Italie. Il met sa bourse et son crédit au service des cardinaux pour le conclave de 1799, mais ses relations seront difficiles avec le cardinal Consalvi, le nouveau secrétaire d'État nommé par Pie VII.

3 Tout aussi intéressantes, les pages consacrées à la vie sociale des Torlonia, à l'éducation de leurs enfants, à leurs familiers et invités, et même à leur lointaine parentèle: à la mort de Joseph Tourlonias, à Aubusson d'Auvergne, Giovanni Torlonia, déjà immensément riche, réclame sa part! Il a acheté le vaste territoire de Roma Vecchia, ferme érigée en marquisat par le pape; en 1803, il acquiert le duché de Bracciano, titre qu'il portera à partir de 1809 et que voyageurs et chroniqueurs mentionnent inévitablement. En 1809, il devient patricien romain, honneur que lui accorde Pie VII 
pour services rendus: il entre donc dans la haute noblesse romaine, aux côtés des Borghese, Colonna, Orsini. En 1814, il est fait prince après l'achat du château et du domaine de Civitella Cesi. Sa quête nobiliaire s'achève en 1820 par l'achat du duché de Poli et Guadagnolo. Sur tous ces châteaux, villas, palais et tombeaux - à Saint-Jean-deLatran, la chapelle funéraire des Torlonia est «décorée comme un café», dit Edmond About -, il appose de très parlantes armes composées d'un bandeau de six roses d'or sur fond bleu parcouru par deux étoiles filantes: sic itur ad astra, aurait dit Coffe! S'il n'est en 1810 qu'au dix-septième rang des plus riches Romains - le prince Borghese caracole en tète avec 2.605810 écus -, en 1820 sa fortune est évaluée à 1082758 écus, dont 85\% en biens immobiliers. À sa mort il laisse un patrimoine de trente- cinq millions d'écus! Ses enfants et petits-enfants vont épouser les rejetons des familles en tête de liste: par exemple, la princesse Anna Maria, unique héritière du colossal patrimoine d'Alessandro Torlonia, épousera le prince Giulio Borghese, lequel devra adopter le patronyme de son épouse pour perpétuer l'illustre nom.

Dans la saga des Torlonia, Giovanni aura pour successeur Alessandro (1800-1886), son fils cadet, le «Rothschild de Rome». Le fils aîné, Marino (1796-1865), moins connu, sera un esthète doublé d'un viveur, héros d'une aventure burlesque que Stendhal mentionne dans une note du manuscrit de Lucien Leuwen; quant à Carlo (1798-1848), il sera le saint homme de la famille et un ami des arts. À noter l'attention que Giovanni porta aux enfants nés d'un premier mariage de sa femme, dont «ce bon Chiaveri», mort du choléra en 1837, que Stendhal apprécia. Alessandro, qui épouse en 1840 Teresa Colonna (ce qui lui permet d'ajouter à ses armoiries la très fameuse colonne), distingué entre tous par son père, fut bien sûr banquier et homme d'affaires, mais aussi collectionneur et mécène (sur cette activité rapidement évoquée, voir Barbara Steindl, Mäzenatentum in Rom des 19 Jahrhunderts. Die Familie Torlonia, 1994). Au nombre de ses opérations, sa grande œuvre, l'assèchement du lac Fucino, auquel César avait déjà pensé et que tenta de réaliser Claude, lui vaudra le titre de prince de Fucino, l'adjudication de la ferme des sels et tabacs que Stendhal mentionne dans ses lettres et rapports à Rigny, Broglie et Guizot. Au vu des services financiers rendus à la papauté, on comprend que Pie VIII l'ait appelé «le père de la patrie» et qu'il ait dit à Anna-Maria Torlonia, épouse de Giovanni: «Votre fils est le mien, il a sauvé l'État»! (propos rapportés par Stendhal à son ministre). Les Torlonia accèderont au rang de prince assistant au trône pontifical, charge qui est encore aujourd'hui dans la famille. Il développe son partenariat avec les Rothschild (caisse d'amortissement de la dette publique, emprunts d'Etat), prend de nombreuses participations (mines, transports, commerce de la laine, etc...). En même temps il agrandit et transforme palais et villas achetés par son père. Les galeries et salons de la véritable reggia qu'est devenu place de Venise le palais Torlonia, ex-palais Bolognetti (démoli au début $\mathrm{du} \mathrm{xx}^{\mathrm{e}}$ siècle), sont ornés de sculptures antiques et d'œuvres d'artistes contemporains (Canova, dont Stendhal admirera le groupe d'Hercule lançant Lycas à la mer, Thorwaldsen, Galli). À cela s'ajoute une collection archéologique décrite par Oliviero Ozzi en 1902 et par Jörgen Hartmann en 1967. La villa Torlonia, via Nomentana, acquise des Colonna en 1797, est restructuré par Giuseppe Valadier de 1802 à 1806, puis par Caretti et Raimondi, sur le modèle de la villa Adriana, avec casino et théâtre. Il y a aussi le palais Torlonia au Borgo (via della Conciliazione), avec ses vastes salons où Alessandro reçut des milliers d'invités et organisa des fêtes mémorables de 1840 à 1845, la villa Albani, achetée en 1868, avec l'ancienne collection Albani, le musée Torlonia de la via della Lungara (constitué à partir de l'ancienne collection Giustiniani, sur les conseils de P.E. Visconti), les théâtres de l'Apollo, de l'Argentina - cédé à la ville 
de Rome - et la salle de l'Alibert. Luxe écrasant de nouveaux riches? Il vaudrait la peine de comparer les Torlonia père et fils à Mayer Amschel Rothschild et à ses cinq fils installés dans les cinq grandes places européennes (Francfort, Londres, Paris, Vienne et Naples - mais pas à Rome!), qui rivalisent de magnificence et dont les collections d'art sont fabuleuses (voir Pauline Prévost-Marcilhacy, Les Rothschild bâtisseurs et mécènes, Flammarion, 1995). G. et A. Torlonia furent à Rome des personnages clés et leur nom est resté quasi-légendaire car, comme le dit le proverbe, «à Dieu et à Torlonia, tout est possible»; ou, mieux encore, Ignazio Silone en 1933: «In capo a tutto c'è Dio, padrone del cielo, poi viene il principe Torlonia, padrone della terra. Poi vengono le guardie del principe, poi vengono i cani delle guardie del principe. Poi il nulla, poi ancora il nulla, poi vengono i cafoni».

5 Dans son épilogue, Henri Ponchon cite opportunément Stendhal, pourtant rien moins que tendre pour les enrichis qui se piquaient de parler littérature, art ou musique: "Quel que soit un homme à millions, en employant les meilleurs sculpteurs et architectes de son siècle, il a une chance d'être immortel». 